\title{
Resultado a largo plazo de la uretroplastia término-terminal
}

\author{
M.A. Ortíz Gorraiz, F.J. Vicente Prados, M. Tallada Buñuel, J.L. Rosales Leal, \\ B. Honrubia Vílchez, A. Fernández Sánchez, F. Vázquez, A. Martínez Morcillo, \\ J.M. Cózar Olmo, E. Espejo Maldonado
}

Hospital Universitario Virgen de las Nieves. Granada.

Actas Urol Esp 2005; 29 (5): 499-505

\section{RESUMEN}

RESULTADOS A LARGO PLAZO DE LA URETROPLASTIA TÉRMINO-TERMINAL

Objetivo: Evaluamos los resultados a largo plazo de la uretroplastia término-terminal.

Material y Métodos: Revisamos 40 pacientes afectos de estenosis de uretra bulbar de etiología diversa: yatrógena $40 \%$, traumática $15 \%$, infecciosa $2 \%$ y desconocida $40 \%$. En 17 casos se realizó previamente uretrotomía interna. El estudio mediante CUMS reveló una localización bulbar en todos los casos y una longitud inferior a 1 cm en 13 casos, entre 1-2 cm en 26 casos y entre $2-3 \mathrm{~cm}$ en 1 caso. El flujo máximo varió de 3-13 ml/s. Se valoró mediante cultivo preoperatorio la ausencia de bacteriuria. El tiempo medio de seguimiento fue de 45 meses (12142 meses). Se consideró resuelta la estenosis cuando no aparecieron datos radiológicos ni flujométricos compatibles con reestenosis.

Resultados: En 37 casos (92\%) los resultados fueron satisfactorios, no precisando los pacientes ningún otro procedimiento quirúrgico secundario. Tras la cirugía el flujo máximo varió entre 18-45 ml/s. En dos de los tres pacientes con reestenosis la uretrotomía interna endoscópica fue resolutiva. Se trató de dos pacientes con estenosis de origen traumático y longitud mayor a 1,5 cm. En el tercer paciente con recidiva se optó finalmente por realizar una nueva uretroplastia termino-terminal, con buen resultado posterior.

Conclusiones: La uretroplastia término-terminal es una técnica altamente resolutiva en la estenosis de uretra bulbar. El diagnóstico preoperatorio se basa en el estudio radiológico (CUMS). El control postoperatorio debe fundamentarse en la clínica y estudio flujométrico. El origen traumático de la estenosis empeora los resultados de la cirugía. En casos de reestenosis la uretrotomía interna endoscópica permite complementar de forma exitosa los resultados de la uretroplastia.

Palabras clave: Estenosis uretral. Uretroplastia. Flujo máximo. Uretrocistografía.

\section{ABSTRACT}

\section{LONG-TERM RESULTS OF END-TO-END URETHROPLASTY}

Objective: We evaluated long term results of end-to-end urethroplasty.

Material and methods: We reviewed 40 patients with bulbar urethral stricture of diverse origin: iatrogenic $40 \%$, traumatic $15 \%$, infectious $2 \%$ and unknown $40 \%$. In 17 cases internal urethrotomy was made previously. The radiological study with retrograde and voiding cystogram revealed a bulbar location in all cases and a length inferior to $1 \mathrm{~cm}$ in 13 cases, between $1-2 \mathrm{~cm}$ in 26 cases and $2-3 \mathrm{~cm}$ in 1 case. The maximum flow rate varied between $3-13 \mathrm{ml} / \mathrm{s}$. The absence of bacteriuria was valued by means of preoperating culture. The average time of pursuit was 45 months (12-142 months). The stricture was considered resolute when not appear compatible radiological or functional finds of failure.

Results: In 37 cases (92\%) the results were satisfactory, without secondary surgical procedure. After surgery maximum flow-rate varied between $18-45 \mathrm{ml} / \mathrm{s}$. In two patients with failure, internal urethrotomy was decisive. In this case the stricture origin was traumatic. The third patient with failure was finally chosen to make new endto-end urethroplasty, with good later result.

Conclusions: End-to-end urethroplasty is a highly decisive technique for bulbar urethral stricture. The preoperating diagnosis is based on the radiological study (retrograde and voiding cystogram). The postoperating control must be based on clinic and uroflow study. Traumatic stricture repair showed worse results. In cases of failure, internal urethrotomy allows to complement successful results of end-to-end urethroplasty. 
$\mathrm{L}$ a estenosis de uretra bulbar es la localización ideal para realizar escisión de la uretra dañada y anastomosis termino-terminal independientemente del origen de la angostura. Los mejores resultados se consiguen en estenosis inferiores a $3 \mathrm{~cm}$ de longitud, no aconsejándose este procedimiento en las de mayor tamaño ${ }^{1}$. Para lograr una curación satisfactoria a largo plazo es fundamental la práctica de una técnica quirúrgica minuciosa, con escisión completa de la zona estenótica (uretra gris) hasta alcanzar uretra sana, y movilización amplia de los cabos uretrales para conseguir una anastomosis libre de tensión que evitará la isquemia de los bordes, causa de fístulas y recurrencia de estenosis posterior. A pesar de cumplir estas premisas adaptadas a cada caso en particular, encontramos pacientes en los que de forma inesperada y sin una evidente causa se produce una nueva estenosis de la anastomosis, recidiva que aun hoy día permite establecer debates en cuanto a su resolución, bien mediante nueva técnica abierta o bien con uretrotomía endoscópica. En cualquier caso, la uretroplastia termino-terminal bien indicada y realizada consigue en las diferentes series publicadas tasas de éxito cercanas al 90\% (Tabla 1).

\section{Tabla 1}

Diferentes series publicadas de uretroplastia término-terminal indicando número de pacientes incluidos, tiempo de seguimiento (meses) y tasa de éxito

\begin{tabular}{cccc}
\hline Estudio & No pacientes & $\begin{array}{c}\text { Seguimiento } \\
\text { (meses) }\end{array}$ & $\begin{array}{c}\text { Éxito } \\
\text { n (\%) }\end{array}$ \\
\hline$[1]$ & 74 & 60 & $66(93 \%)$ \\
{$[2]$} & 24 & 64 & $19(80 \%)$ \\
{$[3]$} & 150 & 44 & $126(84 \%)$ \\
{$[4]$} & 20 & 53 & $19(95 \%)$ \\
{$[5]$} & 32 & 24 & $18(56 \%)$ \\
{$[6]$} & 37 & 60 & $33(89 \%)$ \\
{$[7]$} & 49 & 48 & $47(96 \%)$ \\
Actual & 40 & 45 & $37(92 \%)$ \\
\hline
\end{tabular}

Valoramos nuestra serie de uretroplastia término-terminal, evaluando las características preoperatorias de los casos, así como el resultado final de la cirugía, estableciendo qué casos presentaron fracaso por reestenosis y como se manejaron éstos.

\section{MATERIAL Y MÉTODOS}

Se realizó una revisión retrospectiva de nuestra casuística de 40 pacientes varones afectos de estenosis uretral sometidos entre 1986 y 2004 a uretroplastia término-terminal y cuyo seguimiento se pudo realizar en nuestro Servicio. La edad media de los pacientes fue de 35 años (17-72 años). La etiología de la estenosis se resume en la Tabla 2.

Tabla 2

Distribución de los pacientes según la causa que se estableció para la estenosis

\begin{tabular}{cc}
\hline Etiología & $\begin{array}{c}\text { № pacientes } \\
\text { (\%) }\end{array}$ \\
\hline Yatrógena & $16(40 \%)$ \\
Traumática & $6(15 \%)$ \\
Infecciosa & $2(5 \%)$ \\
Desconocida & $16(40 \%)$ \\
Total & $\mathbf{4 0}$ \\
\hline
\end{tabular}

El estudio diagnóstico preoperatorio se realizó mediante anamnesis detallada, cistouretrografía retrograda y miccional así como uroflujometría aislada cuando el paciente pudo efectuar micción uretral.

En 23 casos se indicó la uretroplastia término-terminal como técnica quirúrgica de primera elección. Los restantes 17 pacientes tenían antecedentes de uretrotomía interna $(n=16)$ y de uretroplastia con colgajo prepucial libre $(n=1)$.

Del estudio radiológico prequirúrgico (CUMS) se estableció que en todos los casos se trataba de estenosis de localización bulbar, con una distribución de la serie en cuanto a longitud de la siguiente forma: inferior a $1 \mathrm{~cm}$ en 13 casos, entre $1-2 \mathrm{~cm}$ en 26 casos y entre $2-3 \mathrm{~cm}$ en 1 caso (Fig. 1).

La valoración del estudio funcional previo a la intervención mediante flujometría arrojó los siguientes datos (valores medios y rango de la serie): volumen miccional $216 \mathrm{ml}$ (107-905 ml); flujo máximo $8,2 \mathrm{ml} / \mathrm{s}(3-13 \mathrm{ml} / \mathrm{s})$; tiempo de flujo $42 \mathrm{~s}$ (29-90 s); tiempo de micción $47 \mathrm{~s}$ (3591 s). Igualmente comprobamos la presentación de la característica morfología en meseta del registro flujométrico en muchos de estos pacientes (Fig. 2). 


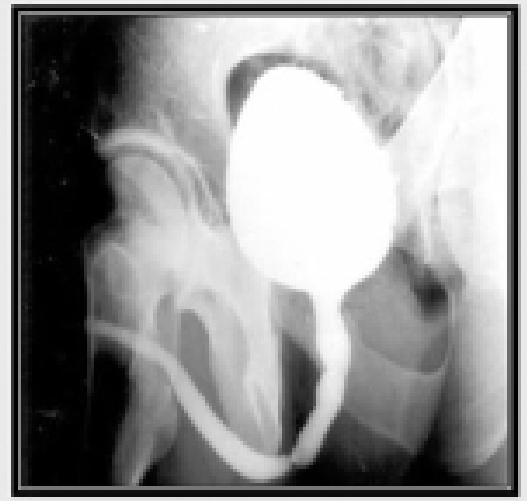

$<1 \mathrm{~cm}(\mathrm{n}=13)$

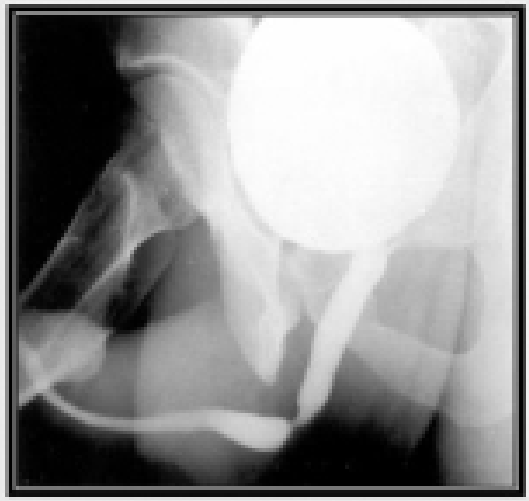

$1-2 \mathrm{~cm}(\mathrm{n}=26)$

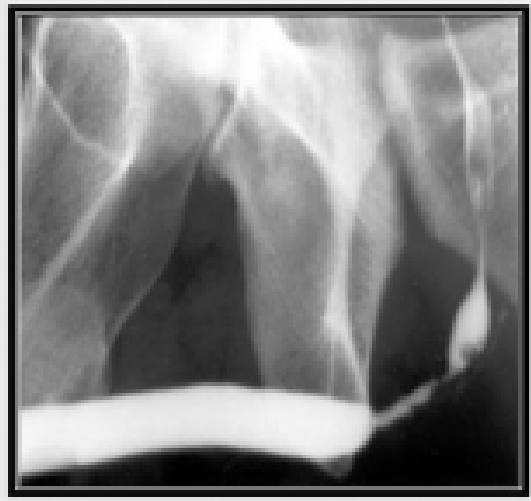

$2-3 \mathrm{~cm}(\mathrm{n}=1)$

FIGURA 1. Distribución de las estenosis en función de su longitud media en el estudio radiológico (CUMS).

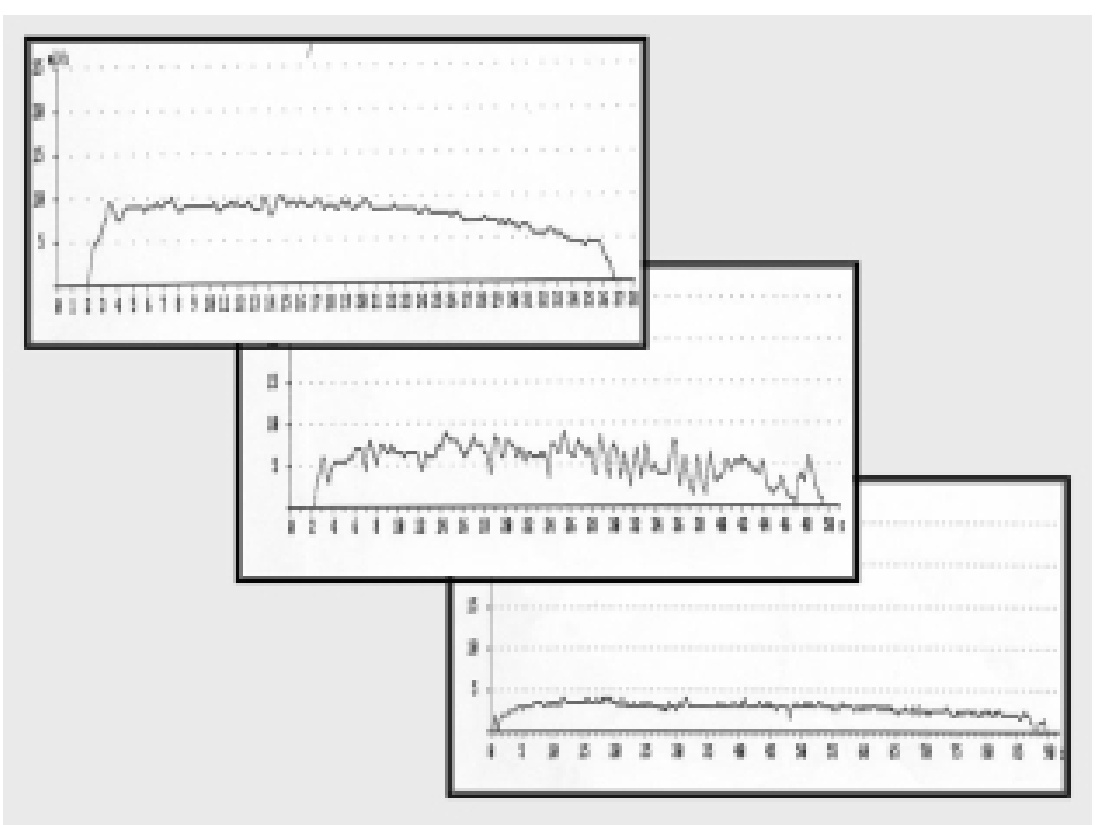

FIGURA 2. Flujometrias pertenecientes a algunos pacientes antes de la uretroplastia. Obsérvese la tipica morfologia en meseta que presentaron las curvas.

Previamente a la intervención a los pacientes se le tomó muestra de orina para urocultivo y antibiograma, pautándose profilaxis antibiótica (Cefuroxima $1500 \mathrm{mg}$ IM) que se mantuvo o modificó tras la cirugía en función de los resultados bacteriológicos, según protocolo propio del Servicio.

$\mathrm{El}$ acceso quirúrgico fue perineal en todos los casos, con el paciente colocado en posición de litotomía exagerada. En tres pacientes con estenosis de origen traumático y portadores de derivación vesical suprapúbica en los cuales se había diferido la intervención, se realizó un acceso combinado perineal-hipogástrico para identificar más correctamente el cabo estenótico proximal mediante exploración anterógrada.

La técnica quirúrgica se basó en la liberación amplia de la uretra, con buena movilización de ésta, posterior escisión "en pico de pato" de la zona estenótica con espongiofibrosis hasta alcanzar uretra sana. Para aumentar la luz uretral se espatularon ambos cabos uretrales, colocando tutor uretral y realizando anastomosis uretral termino-terminal con sutura discontinua reabsorbible en monoplano. Se colocó drenaje aspirativo perineal en todos los casos $(24 \mathrm{~h})$. La duración media de la intervención fue de $117 \mathrm{mi}$ nutos. La estancia media de los pacientes fue de 2,8 días.

El tutor utretral se mantuvo aproximadamente dos semanas, retirándose en este tiempo bajo control radiológico de la anastomosis. Solamente en dos casos fue necesario mantener el tutor uretral una semana más por evidencia de extravasado a nivel de la anastomosis a los 14 días de la intervención.

El tiempo medio de seguimiento en esta serie fue de 45 meses (12-142 meses), realizándose en las valoraciones posquirúrgicas estudio clínico y 
flujométrico, con control radiológico ante la sospecha de reestenosis. Las revisiones se realizaron a los 3 meses de la cirugía y posteriormente semestralmente los 3 primeros años, con revisión anual si no existían problemas a partir de los 3 años. Se consideró resuelta la estenosis cuando no existían datos radiológicos (Fig. 3) ni flujométricos $(\mathrm{Qmax} \geq 15 \mathrm{ml} / \mathrm{s})$ compatibles con reestenosis.

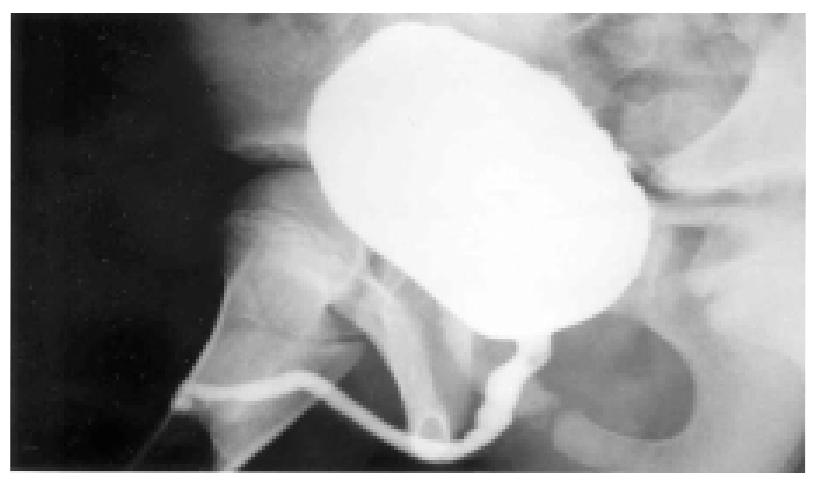

FIGURA 3. Cistouretrografia miccional de control al año de la uretroplastia donde se aprecia un adecuado calibre uretral asi como la ausencia de estenosis.

\section{RESULTADOS}

En 37 casos (92\%) los resultados fueron satisfactorios para un seguimiento medio cercano a los 4 años, con resolución definitiva de la estenosis según los criterios mencionados anteriormente sin precisar los pacientes ningún otro procedimiento quirúrgico secundario tras la uretroplastia termino-terminal.

Las complicaciones derivadas de la cirugía fueron 2 casos de infección de herida y 1 hematoma perineal, que se resolvieron con medidas conservadoras y que no motivaron fallo posterior de la uretroplastia.

Desde el punto de vista funcional, el estudio flujométrico posquirúrgico (Figura 4) de esta serie de pacientes mostró un incremento considerable
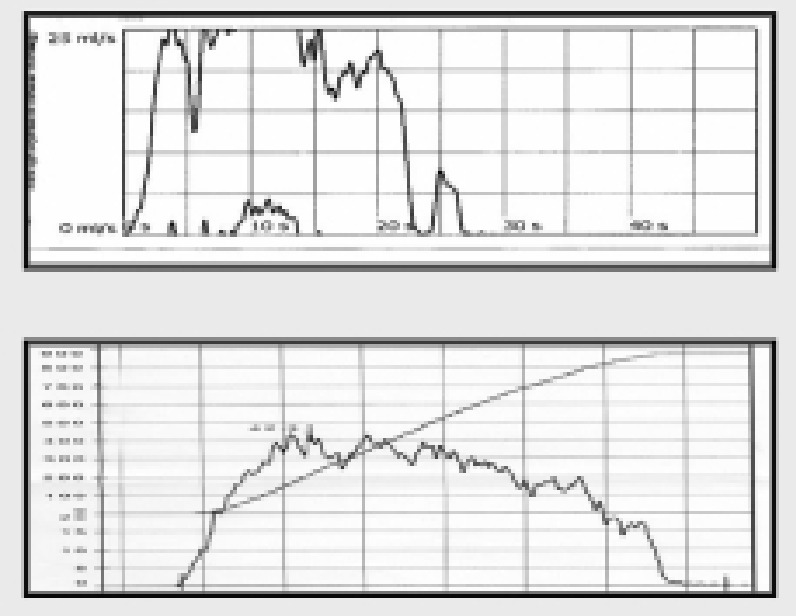

FIGURA 4. Varios ejemplos de flujometria aislada de control tras la uretroplastia que muestran morfologia y flujos compatibles con la normalidad.

del flujo máximo, con una media de $29 \mathrm{ml} / \mathrm{s}$ y un rango de 18-45 ml/s. En pacientes con clínica compatible de reestenosis o flujo obstructivo se realizó CUMS de control.

En tres pacientes se produjo una recidiva de la estenosis a nivel de la anastomosis (Tabla 3). El tiempo medio hasta la reestenosis fue de 13 meses (8-24 meses). En dos casos el origen de la estenosis primaria fue traumático y la longitud de ésta fue de $1^{`} 7$ y $2^{`} 2 \mathrm{~cm}$, optándose en ambos pacientes por la realización de uretrotomía interna endoscópica por tratarse de estrecheces de muy corta extensión, no siendo preciso repetir ésta. En ambos casos no se ha presentado recidiva posterior. El tercer caso de recidiva se trató de un varón joven con estenosis primaria sin causa conocida y longitud inferior a $1 \mathrm{~cm}$. Este paciente ha requerido la realización de un total de dos uretrotomías endoscópicas y una nueva uretroplastia termino-terminal para el control de su patología, con buena evolución en la actualidad tras doce meses de seguimiento.

\section{Tabla 3}

Pacientes en los que fracasó la cirugía. Se indican la etiología de la estenosis primaria así como su longitud y tiempo hasta reestenosis, señalando la técnica quirúrgica empleada para su resolución

\begin{tabular}{ccccc}
\hline Paciente & Etiología & Longitud & Tiempo hasta reestenosis & Procedimiento \\
\hline 1 & Traumática & $2,2 \mathrm{~cm}$ & 8 meses & Uretrotomía interna \\
2 & Traumática & $1,4 \mathrm{~cm}$ & 14 meses & Uretrotomía interna \\
3 & Desconocida & $0,7 \mathrm{~cm}$ & 24 meses & Uretroplastia T-T \\
\hline
\end{tabular}




\section{DISCUSIÓN}

En las diferentes series publicadas de estenosis de uretra sometida a uretroplastia terminoterminal los resultados coinciden en señalar una alta tasa de éxito cercana al $90 \%$, por lo que nuestros datos estarían en consonancia con la bibliografia revisada (Tabla 1).

Un primer punto de discusión que se nos plantea al valorar pacientes con estenosis de uretra que van a ser sometidos a tratamiento quirúrgico es la técnica de primera elección para cada uno de ellos. Aunque para algunos autores $^{\mathbf{1}}$ la presencia de una estenosis corta inferior al centímetro puede ser indicación de hasta dos uretrotomías internas antes de intentar la uretroplastia término-terminal, nosotros preferimos dejar la uretrotomía interna como técnica de elección en pacientes que bien presenten una estenosis anular única de escasos milímetros, o bien estenosis más complejas en pacientes con alto riesgo quirúrgico. Optamos por ir directamente a la uretroplastia términoterminal en pacientes jóvenes, con estenosis nunca mayores de $3 \mathrm{~cm}$, y en los que sabemos que la realización de una técnica endoscópica no va a ser resolutiva total. Entre estos dos extremos estaría el grupo, posiblemente mayor en número, de pacientes con características intermedias en los cuales la decisión puede ser más difícil a la hora de optar por la mejor técnica, ya que si bien es conocida la alta tasa de recidivas en la cirugía endoscópica, con tasas de recidiva cercanas al $70 \%$ en seguimiento a largo plazo $^{8}$ no es menos cierto que se trata de una intervención rápida y exenta prácticamente de complicaciones. Otro dato a favor de realizar la uretroplastia termino-terminal como cirugía de primera elección es la idea propugnada por algunos autores ${ }^{2,3}$ de que la realización de múltiples uretrotomías endoscópicas en una estenosis recidivante puede motivar un mayor grado de espongiofibrosis con una posterior dificultad añadida al realizar una técnica abierta y un posible peor resultado a largo plazo. Este dato, recogido en algunas series publicadas ${ }^{2,3}$, no lo hemos comprobado en la nuestra, de forma que no encontramos peores resultados en los pacientes que presentaban antecedentes de uretrotomía interna. Esto podría ser explicado por el hecho que los casos presentados sólo se habían sometido a una cirugía endoscópica previa a la uretroplastia.

Destacamos del apartado de resultados la escasa morbilidad de la técnica quirúrgica, presentándose en esta serie un $5 \%(n=2)$ de infecciones de herida y en un 2,5\% $(n=1)$ hematoma perineal, que no motivaron fracaso posterior de la técnica quirúrgica. Estas cifras han sido similares en otras series ${ }^{2,3}$. Otra complicación poco comunicada, y que debemos tener en cuenta es la presentación en el postoperatorio inmediato del sindrome compartimental, cuadro grave derivado de la posición de litotomía exagerada y que si no es reconocido a tiempo puede tener graves consecuencias para el paciente ${ }^{9}$. De forma tardía se han descrito encordamiento peneano en resecciones amplias de uretra e incontinencia de orina o impotencia, siendo estas dos últimas en casos de afectación de uretra posterior ${ }^{3}$. En nuestro trabajo, por tratarse de una revisión retrospectiva, no pudimos valorar la función sexual previa de los pacientes, por lo que no se incluyeron datos acerca de la esfera sexual tras la cirugía.

Otro punto que nos parece interesante es el tipo de tutor uretral a utilizar y el tiempo que debe mantenerse tras la cirugía. Nosotros preferimos una sonda uretral acanalada que permite la expulsión perisonda del exudado uretral, reduciendo el riesgo de infecciones por retención de este exudado con posterior formación de fístulas. Igualmente hemos comprobado que es suficiente con mantener el tutor durante dos semanas, ya que mediante control radiológico solo hemos evidenciado dos casos de extravasado a nivel de la anastomosis que se resolvió sin incidencias manteniendo la sonda una semana más. En la bibliografía revisada, los tiempo de sondaje uretral variaron entre los 7 y 21 días ${ }^{1,5}$.

En relación con las pruebas diagnósticas pensamos que una anamnesis detallada recogiendo antecedentes de interés y sintomatología puede ser muy orientativa en casos de estenosis de uretra, complementada con el estudio radiográfico mediante CUMS que valorará localización, longitud y grado de estenosis, si bien es cierto que en función de la proyección puede generalmente infravalorar el tamaño de ésta. La realización de examen uretroscópico no suele ser necesario y 
más bien huimos de él por tratarse de una prueba invasiva no exenta de riesgos en estos pacientes. El estudio flujométrico, que puede completar el estudio diagnóstico, tendrá mayor interés en el seguimiento de estos pacientes que en el propio diagnóstico inicial, ya que puede de forma objetiva valorar la calidad de la micción tras la intervención. En nuestra serie fue muy significativa la diferencia de los valores del flujo máximo, pasando de $8,7 \mathrm{ml} / \mathrm{s}$ previo a la cirugía a $29 \mathrm{ml} / \mathrm{s}$ tras la intervención. En los casos que cae de forma importante el flujo miccional, y ante la sospecha de reestenosis, es preceptiva la realización de nuevos estudio radiológicos para valorar esta posibilidad.

Algunos autores han realizado estudios para determinar la utilidad de los ultrasonidos en el diagnóstico de la estenosis de uretra mediante el empleo de potenciadores sonográficos. Morey y cols. han valorado preoperatoriamente la longitud y grado de estenosis uretral, así como la intensidad de la espongiofibrosis asociada a la zona estenótica, con gran exactitud en los resultados obtenidos ${ }^{10}$. En este artículo no mostramos datos propios al tratarse de una técnica no implantada en nuestro centro.

La relación entre etiología de la estenosis y fracaso de la técnica ha sido señalada por algunos autores ${ }^{3,11}$, estableciendo peores resultados quirúrgicos en estenosis postraumáticas, si bien las tasas de éxito tras una segunda cirugía han mostrado resultados favorables con desaparición de la reestenosis hasta en el $60-87 \%$ de los casos $^{11-13}$. En nuestra serie, de igual forma, encontramos una frecuencia de recidiva mayor en pacientes con estenosis primaria de etiología traumática $(2 / 6$ pacientes, $33 \%)$ si bien fue resuelta mediante uretrotomía posterior en ambos casos. De forma general sabemos que los factores de mal pronóstico en pacientes con estenosis de uretra en los que se plantee la uretroplastia termino-terminal podrían ser: longitud excesiva de la estenosis (mayor de $3 \mathrm{~cm}$ ), origen traumático, uretra cicatricial en relación o no con cirugías uretrales previas, presencia de fístula o de divertículo uretral, gran afectación del tejido periuretral y existencia de infección urinaria activa en el momento de la intervención ${ }^{3,5}$. Ésta última, en nuestra opinión, tiene gran importancia, de forma que consideramos fundamental erradicar la infección urinaria previa a la cirugía, para lo cual se ha desarrollado un protocolo de manejo quirúrgico que permite que el paciente sea tratado previamente a la cirugía de una bacteriuria significativa, tanto sintomática como asintomática, mediante la toma de cultivo unas dos semanas antes de la intervención, confirmando al ingreso su resolución mediante nuevo urocultivo de control.

No se ha establecido el tiempo de seguimiento preciso para determinar la curación definitiva de la estenosis, si bien se sabe que el éxito de la técnica disminuye con el tiempo y se han descrito recurrencias incluso a los 10 años de la cirugía 4 . Se requiere por tanto estudios a muy largo plazo para establecer la verdadera tasa de éxito de la uretroplastia término-terminal ya que la mayoría de trabajos establecen un tiempo medio de seguimiento alrededor de los 40-50 meses.

Para concluir señalaremos el papel de la cirugía endoscópica en el tratamiento de la estenosis a nivel de la anastomosis tras uretroplastia terminoterminal. Pensamos que la uretrotomía es la técnica ideal y complementaria en la mayoría de estos casos al tratarse en muchas ocasiones de estrecheces de pocos milímetros en las que la uretrotomía permite generalmente de forma sencilla y rápida resolver el fracaso tras cirugía abierta uretral, permitiendo alcanzar mediante ambas técnicas combinadas altas tasas de éxito $197 \%$ en nuestra serie). En casos más complejos con reestenosis de mayor longitud se podría intentar de nuevo uretroplastia término-terminal siempre que no se produzca un acortamiento importante del pene, o bien recurrir a técnicas de sustitución uretral mediante colgajos genitales o extragenitales ${ }^{14}$.

En un futuro, las técnicas quirúrgicas aplicadas a la estenosis de uretra deberán enfrentarse a nuevos arsenales terapéuticos derivados del conocimiento cada vez más profundo de la patogenia de este cuadro en relación con los cambios histológicos que ocurren en el tejido a nivel de su matriz extracelular y composición de ésta ${ }^{15}$.

\section{CONCLUSIONES}

La uretroplastia término-terminal es una intervención definitiva que indicada correctamente y ejecutada de forma reglada consigue tasas de éxito 
del $90 \%$ a largo plazo, con una baja incidencia de morbilidad asociada, de forma que debe ser tenida en cuenta como técnica de primera elección en estenosis cortas y únicas de la uretra bulbar o bulbo-membranosa, sobre todo en pacientes jóvenes. El diagnóstico de la estenosis de uretra se basa fundamentalmente en el estudio radiológico mediante cistouretrografía miccional y retrograda. Pensamos que es fundamental la ausencia de bacteriuria en el momento de la intervención, por lo que defendemos el control bacteriológico previo y profilaxis antibiótica mantenida tras la cirugía. En el seguimiento, sin embargo, será la flujometría aislada la prueba que nos permitirá de forma no invasiva controlar la evolución del paciente, pudiendo establecer que casos requerirán un estudio radiológico posquirúrgico si se presenta de nuevo un flujo bajo, con el fin de diagnosticar posibles reestenosis de la anastomosis. El origen traumático de la estenosis supone un factor de mal pronóstico a la hora de valorar los resultados obtenidos con esta técnica. El manejo de la obstrucción posquirúrgica tras la uretroplastia mediante uretrotomía interna endoscópica es una alternativa válida que puede complementar de forma eficaz los buenos resultados de la cirugía abierta, consiguiendo entre ambas un importante beneficio terapéutico en la estenosis de uretra.

\section{REFERENCIAS}

1. Micheli E, Ranieri A, Peracchia G, Lembo A. End-to-end urethroplasty: long term results. BJU Int 2002; 90:68-71.

2. Martínez Carrancio L, Garimaldi Pérez C, Cabezas Checci C, Fernández García ML, Zungri Telo E. Estenosis de la uretra bulbo membranosa: nuestra experiencia en uretroplastia termino-terminal. Actas Urol Esp 1998; 22:350-354.

3. Martínez-Piñeiro JA, Cárcamo $\mathrm{P}$, García Matres $\mathrm{MJ}$, Mártinez-Piñeiro L, Robles Iglesias J, Rodríguez Ledesma JM. Escisión and anastomotic repair for urethral stricture disease: experience with 150 cases. Eur Urol 1997; 32:433441 .
4. Barbagli G, Palminteri E, Bartoletti R, Selli C, Rizzo M. Long-term results of anterior and posterior urethroplasty with actuarial evaluation of the succes rates. J Urol 1997; 158:1380-1382.

5. Roehborn CG, McDonnell JD. Analysis of factors contributing to succes or failure of 1-stage urethroplasty for urethral stricture disease. J Urol 1994; 151:869-874.

6. Mundy AR. Results and complications of urethroplasty and its future. Br J Urol 1993; 71:322-325.

7. Lindell O, Bordowski J, Noll F, Schreiter F. Urethral stricture repair: results in 179 patients. Scand J Urol Nephrol 1993; 27:241-245.

8. Pensadoro V, Emilozzi P. Internal urethrotomy in the management of anterior urethral strictures: long term follow-up. J Urol 1996;156:73-75.

9. Santiago FM, Ortiz M, Linde C, Colina L, Molina M, Santiago J. Sindrome compartimental bilateral como complicación de la posición de litotomía dorsal prolongada. Rev Esp Anestesiol Reanim 2004; 51:176-177.

10. Morey AF, McAninch JW. Ultrasound evaluation of the male urethra for assessment of urethral stricture. J Clin Ultrasound 1996, 24:473-479.

11. Wadhwa SN, Chahal R, Hemal AK, Gupta NP, Dogra PN, Seth A. Management of obliterative posttraumatic posterior urethral strictures after failed initial urethroplasty. J Urol 1998, 159:1898-1902.

12. Barbagli G, Selli C, Tosto A. Reoperative surgery for recurrent strictures of the penile and bulbous urethra. J Urol 1996, 156:76-77.

13. Al-Rifaei MA, Al-Rifaei A. Management of postoperative obstruction after bulboprostatic anastomotic urethroplasty for membranous urethral defects secondary to pelvic fracture. Scand J Urol Nephrol 2001, 35:491-496.

14. Cózar Olmo JM, Moreno J, Espejo Maldonado E, Tallada Buñuel M. Tratamiento quirúrgico de la estenosis uretral. En: Obstrucción del aparato urinario inferior del adulto. Ponencia Oficial al VI Congreso de la Asociación Andaluza de Urología (1993).

15. Da-Silva EA, Sampaio FJB, Dornas MC, Damiao R, Cardoso LEM. Extracellular matriz changes in urethral stricture disease. J Urol 2002, 168:805-807.

Dr. M.A. Ortiz Gorraiz

Servicio de Urología

Hospital Universitario Virgen de las Nieves

Avda. de las Fuerzas Armadas, 2

18014 Granada

(Trabajo recibido el 5 marzo de 2005) 